TOKYO J. MATH.

Vol. 1 , No. 2,1978

\title{
More on the Schur Index and the Order and Exponent of a Finite Group
}

\author{
Toshihiko YAMADA \\ Tokyo Metropolitan University
}

Let $G$ be a finite group and $K$ a field of characteristic 0 . Let $\chi$ be an absolutely irreducible character of $G$ and let $m_{K}(\chi)$ denote the Schur index of $\chi$ over $K$. In Fein and Yamada [1], we gave a theorem which relates $m_{\boldsymbol{Q}}(\chi)$ to the order and exponent of $G$, where $\boldsymbol{Q}$ is the rational field. In this paper, we will give similar results for the case $K=\boldsymbol{Q}_{l}$, the $l$-adic numbers, where $l$ is a prime. These results are easily derived from the formula of index of an $l$-adic cyclotomic algebra, which was obtained by the author [4], [5].

For the rest of the paper, $k$ is a cyclotomic extension of $\boldsymbol{Q}_{l}$, i.e., $k$ is a subfield of a cyclotomic field $\boldsymbol{Q}_{l}\left(\zeta^{\prime}\right)$, where $\zeta^{\prime}$ is a root of unity. For a natural number $n, \zeta_{n}$ denotes a primitive $n$-th root of unity. A cyclotomic algebra over $k$ is a crossed product

$$
\begin{gathered}
B=(\beta, k(\zeta) / k)=\sum_{\sigma \in \zeta} k(\zeta) u_{\sigma}, \quad\left(u_{1}=1\right), \\
u_{\sigma} x=\sigma(x) u_{\sigma} \quad(x \in k(\zeta)), \quad u_{\sigma} u_{\tau}=\beta(\sigma, \tau) u_{\sigma \tau}, \quad(\sigma, \tau \in \mathscr{G}),
\end{gathered}
$$

where $\zeta$ is a root of unity, $\mathscr{G}$ is the Galois group of $k(\zeta)$ over $k$, and $\beta$ is a factor set whose values are roots of unity in $k(\zeta)$. Put $L=k(\zeta)$. Let $\varepsilon(L)$ denote the group of roots of unity contained in $L$. Let $\varepsilon^{\prime}(L)$ (respectively, $\varepsilon_{l}(L)$ ) denote the subgroup of $\varepsilon(L)$ consisting of those roots of unity in $L$ whose orders are relatively prime to $l$ (respectively, powers of $l$ ). We have $\varepsilon(L)=\varepsilon^{\prime}(L) \times \varepsilon_{l}(L)$. Let

$$
\beta(\sigma, \tau)=\alpha(\sigma, \tau) \gamma(\sigma, \tau), \quad \alpha(\sigma, \tau) \in \varepsilon^{\prime}(L), \quad \gamma(\sigma, \tau) \in \varepsilon_{l}(L) .
$$

Suppose that $l$ is an odd prime. Let $\langle\theta\rangle$ denote the inertia group and $\phi$ a Frobenius automorphism of the extension $k(\zeta) / k$. The order $e$ of $\theta$ has the form $e=l^{t} e^{\prime}, e^{\prime} \mid l-1$. Let $f$ denote the residue class degree of the extension $k / Q_{l}$, so $\zeta_{l} f_{-1} \in k$. 
THEOREM 1 (Yamada [4]). Let $l$ be an odd prime and $k$ a cyclotomic extension of $\boldsymbol{Q}_{l}$. Notation being as above, let $(\beta, k(\zeta) / k) \sim(\alpha, k(\zeta) / k) \bigotimes_{k}$ $(\gamma, k(\zeta) / k)$ be a cyclotomic algebra over $k$ given by (1)-(3). Then the number

$$
\delta=(\alpha(\theta, \phi) / \alpha(\phi, \theta))^{e /\left(l^{f}-1\right)} \alpha(\theta, \theta) \alpha\left(\theta^{2}, \theta\right) \cdots \alpha\left(\theta^{e-1}, \theta\right)
$$

belongs to $k$, so that we can write $\delta=\zeta_{l}^{0} f_{-1}$ for a certain integer $v$. The index of the cyclotomic algebra $(\beta, k(\zeta) / k)$ is equal to $e^{\prime} /\left(v, e^{\prime}\right)$.

Proof. In [4, Theorem 3], this theorem is stated for the case $k(\zeta)=Q_{l}\left(\zeta^{\prime}\right), \zeta^{\prime}$ being some root of unity. But it is easy to see that the same proof is also valid for any extension $k(\zeta) / k, \zeta$ being a root of unity.

CoROLlary 2. Notation being as in Theorem 1, suppose that the factor set $\beta$ has all its values equal to roots of unity of order prime to $l$, i.e., $\beta(\sigma, \tau) \in \varepsilon^{\prime}(k(\zeta))$, for all $\sigma, \tau \in \mathscr{G}$. Furthermore, suppose that $e=e^{\prime}$, i.e., the ramification index $e$ of the extension $k(\zeta) / k$ is not divisible by $l$. Then the index of the l-adic cyclotomic algebra $(\beta, k(\zeta) / k)=\sum_{o} k(\zeta) u_{o}$ divides the least common multiple of the orders of the elements $\left[u_{\theta}, u_{\phi}\right]$ and $u_{j}^{l^{f}-1}$, where $\left[u_{\theta}, u_{\phi}\right]=u_{\theta} u_{\phi} u_{\theta}^{-1} u_{\phi}^{-1}$.

Proof. We have $\beta(\sigma, \tau)=\alpha(\sigma, \tau), \gamma(\sigma, \tau)=1$ for any $\sigma, \tau \in \mathscr{G}$. Since $\left[u_{\theta}, u_{\phi}\right]=\beta(\theta, \phi) / \beta(\phi, \theta)$ and $u_{\theta}^{s}=\beta(\theta, \theta) \beta\left(\theta^{2}, \theta\right) \cdots \beta\left(\theta^{*-1}, \theta\right)$, it follows that $\left[u_{\theta}, u_{\phi}\right]$ and $u_{\theta}^{\prime}$ commute. Since $e=e^{\prime}$ and $e^{\prime} \mid l-1$, then

$$
\begin{aligned}
\delta^{\left(l^{f} f\right) / e} & =(\beta(\theta, \phi) / \beta(\phi, \theta)) \cdot\left\{\beta(\theta, \theta) \beta\left(\theta^{2}, \theta\right) \cdots \beta\left(\theta^{e-1}, \theta\right)\right\}^{\left(l^{f}-1\right) / e} \\
& =\left[u_{\theta}, u_{\phi}\right] \cdot\left(u_{\theta}^{e}\right)^{\left(l^{f}-1\right) / e}=\left[u_{\theta}, u_{\phi}\right] \cdot u_{\theta}^{l^{f}-1} .
\end{aligned}
$$

Moreover, $\left[u_{\theta}, u_{\phi}\right]$ and $u_{\theta}^{2 f-1}$ commute. On the other hand,

$$
\delta^{\left(l_{-1}^{f}\right) / e}=\zeta_{l f-1}^{v\left(f_{-1}^{f}\right) / e}=\zeta_{\theta}^{v},
$$

whose order is equal to $e /(v, e)=e^{\prime} /\left(v, e^{\prime}\right)$, the index of $(\beta, k(\zeta) / k)$. The corollary now follows at once.

THEOREM 3. Let $G$ be a finite group and $\chi$ an absolutely irreducible character of $G$. Suppose that $l$ is an odd prime and $p$ is a prime such that $p^{n} \neq 1$ divides the Schur index $m_{Q_{l}}(\chi)$ but $p^{n+1}$ does not divide $m_{Q_{l}}(\chi)$. Then either $p^{2 n}$ divides the exponent of $G$ or $p^{n}$ divides the exponent of $G^{\prime}$, the commutator subgroup of $G$, and if $p^{2 n}$ does not divide the exponent of $G$ then $p^{2 n+1}$ divides the order of $G$. If a Sylow p-subgroup of $G$ is abelian, then $p^{2 n}$ divides the exponent of $G$.

Proof. By Theorem $1, p^{n} \mid l-1$. Let $s$ be the exponent of $G$ and 
let $k$ be the subfield of $\boldsymbol{Q}_{l}\left(\zeta_{s}\right)$ such that $\boldsymbol{Q}_{l}\left(\zeta_{s}\right) \supset k \supset \boldsymbol{Q}_{l}(\chi),\left[\boldsymbol{Q}_{l}\left(\zeta_{s}\right): k\right]$ is a power of $p$ and $p \nmid\left[k: \boldsymbol{Q}_{l}(\chi)\right]$. By the Brauer-Witt theorem (see [6, p. 31]) there is a hyperelementary subgroup $H$ (at $p)$ of $G$ and an irreducible character $\xi$ of $H$ with the following properties: (1) there is a normal subgroup $N$ of $H$ and a linear character $\psi$ of $N$ such that $\xi=\psi^{H}$; (2) $H / N \cong \mathscr{G}=\operatorname{Gal}(k(\psi) / k) ;(3) k(\xi)=k ;(4) m_{k}(\xi)=p^{n}$; (5) for every $h \in H$ there is a $\tau(h) \in \mathscr{G}$ such that $\psi\left(h n h^{-1}\right)=\tau(h)(\psi(n))$ for all $n \in N$; and (6) the simple component $A(\xi, k)$ of the group algebra $k[H]$ corresponding to $\xi$ is isomorphic to the cyclotomic algebra $(\beta, k(\psi) / k)=\sum_{\tau \in S} k(\psi) u_{\tau}$ where, if $D$ is a complete set of coset representatives of $N$ in $H(1 \in D)$ with $h h^{\prime}=n\left(h, h^{\prime}\right) h^{\prime \prime}$ for $h, h^{\prime}, h^{\prime \prime} \in D, n\left(h, h^{\prime}\right) \in N$, then $\beta\left(\tau(h), \tau\left(h^{\prime}\right)\right)=\psi\left(n\left(h, h^{\prime}\right)\right)$. Since $\boldsymbol{Q}_{l}\left(\zeta_{s}\right) \supset k(\psi) \supset k$ and $[H: N]=[k(\psi): k]$ is a power of $p$, we may assume that $D$ is contained in a Sylow $p$-subgroup of $H$, and so for any $\tau, \tau^{\prime} \in$ $\mathscr{G}, \beta\left(\tau, \tau^{\prime}\right)$ is a root of unity whose order is a power of $p$. In particular, the factor set $\beta$ has all its values equal to roots of unity of order prime to $l$.

Let $N_{0}$ be the kernel of $\psi$ and $\zeta$ a primitive $\left|N / N_{0}\right|$-th root of unity. Then $k(\psi)=k(\zeta)$ and $N_{0}$ is also the kernel of $\xi$. Moreover, the cyclotomic algebra $(\beta, k(\zeta) / k)=\sum_{\tau} k(\zeta) u_{\tau}$ contains the finite group $F=\left\langle\zeta, u_{\tau}(\tau \in \mathscr{G})\right\rangle$, which is canonically isomorphic to $H / N_{0}$, i.e., $F$ is a section of $G$.

Let $\langle\theta\rangle$ denote the inertia group and $\phi$ a Frobenius automorphism of the extension $k(\zeta) / k$. Let $f$ be the residue class degree of $k / Q_{l}$. The order of $\langle\theta\rangle$ is a power of $p$, so is relatively prime to $l$. Corollary 2 now yields that $p^{n}$, the index of $(\beta, k(\zeta) / k)$, divides the least common multiple of the orders of the elements $\left[u_{\theta}, u_{\phi}\right]$ and $u_{\theta}^{l^{f}-1}$ of $F$. Hence either $p^{n}$ divides the exponent of $F^{\prime \prime}$ or $p^{2 n}$ divides the exponent of $F$, because $l^{f}-1 \equiv l-1 \equiv 0\left(\bmod p^{n}\right)$. If a Sylow $p$-subgroup of $G$ is abelian, then a Sylow $p$-subgroup of $H$ is also abelian, and so $h h^{\prime}=h^{\prime} h$ for any $h, h^{\prime} \in D$. By the isomorphism $H / N_{0} \cong F$, this implies $u_{\tau} u_{\tau^{\prime}}=u_{\tau^{\prime}} u_{\tau}$ for any $\tau, \tau^{\prime} \in \mathscr{G}$. In particular, $\left[u_{\theta}, u_{\phi}\right]=1$, and consequently, $p^{2 n}$ divides the order of $F$.

If $p^{2 n}$ does not divide the exponent of $F$, then $p^{n}$ divides the order of $\left[u_{\theta}, u_{\phi}\right] \in\langle\zeta\rangle$, so $p^{n}||\langle\zeta\rangle \mid$. Recall that $F=\left\langle\zeta, u_{\theta}, u_{\phi}\right\rangle D\langle\zeta\rangle$ and $F /\langle\zeta\rangle \cong$ $\langle\theta, \phi\rangle=\mathscr{G}$. By Theorem 1, $p^{n}$ divides the order of $\theta$, so $p^{n+1}$ divides $[F:\langle\zeta\rangle]$. Hence $p^{2 n+1}|| F \mid$. Since $F$ is a section of $G$, Theorem 3 is proved.

Next we will give a corresponding result for the 2 -adic number field $\boldsymbol{Q}_{2}$. It is known that $m_{Q_{2}}(\chi)=1$ or 2 for any irreducible character $\chi$ of a finite group $G$.

THEOREM 4. Let $G$ be a finite group and $\chi$ an irreducible character 
of $G$. If $m_{Q_{2}}(\chi)=2$, then $2^{2}$ divides the exponent of $G, 2$ divides the exponent of $G^{\prime}$, and $2^{3}$ divides the order of $G$.

PRoof. As in the proof of Theorem 3, the Brauer-Witt theorem implies that there is a 2-adic cyclotomic algebra $B=(\beta, k(\zeta) / k)=\sum_{\tau \in \zeta} k(\zeta) u_{\tau}$, $\mathscr{G}=\operatorname{Gal}(k(\zeta) / k)$, with the following properties: $(1) \zeta$ is a root of unity and $k$ is a cyclotomic extension of $Q_{2} ;(2)$ the index of $B$ equals 2; (3) if $\zeta$ has order $2^{t} r,(2, r)=1$, then $\beta(\sigma, \tau) \in\left\langle\zeta_{2}\right\rangle$ for $\sigma, \tau \in \mathscr{G}$; (4) $B$ contains a finite group $F=\left\langle\zeta, u_{\tau}(\tau \in \mathscr{G})\right\rangle$, which is isomorphic to a section of $G$; (5) $F \triangleright\langle\zeta\rangle$ and $F /\langle\zeta\rangle \cong \mathscr{G}$.

Since $B$ has index 2, then $\zeta_{4} \notin k$ (see [3, Satz 12] or [5, Proposition 5.4]). Furthermore, $t \geqq 2$, because if $t \leqq 1$, then $k(\zeta) / k$ would be unramified and the index of $B$ would be equal to 1 . Hence $2^{2}$ divides the exponent of $F$. By Theorem 3.1 of [5], we see easily that $\mathscr{G}$ contains an automorphism $\iota$ with $\iota\left(\zeta_{2} t\right)=\zeta_{2^{t}}^{-1}$. Then $u_{\iota} \zeta_{2} t u_{\iota}^{-1}=\zeta_{t^{t}}{ }^{1}$ and the commutator $\left[u_{\iota}, \zeta_{2} t\right]=$ $\zeta_{2^{t}} t^{2} \in F^{\prime}$ has order $2^{t-1} \geqq 2$, i.e., 2||$F^{\prime} \mid$. Since $\iota \in \mathscr{G}$ has order 2 , then $|F|=$ $[F:\langle\zeta\rangle] \cdot|\langle\zeta\rangle|=|\mathscr{G}| \cdot|\langle\zeta\rangle| \equiv 0(\bmod 8)$, as was to be shown.

Let $\boldsymbol{R}$ be the real numbers. Let $G$ be a finite group and $\chi$ an irreducible character of $G$. Although $m_{R}(\chi)=1$ or 2 , Theorem 4 does not necessarily hold for the case $m_{R}(\chi)=2$. We will give such an example.

REMARK. Let $G=\langle a, b\rangle$ be the group of order 12 with the defining relations $a^{6}=1, b^{2}=a^{3}, b a b^{-1}=a^{-1}$. Then $|G|=$ exponent of $G=2^{2} 3,\left|G^{\prime}\right|=3$. It is easy to see that $G$ has a faithful irreducible character $\chi$ which is induced from a faithful linear character $\psi$ of $\langle a\rangle$. The simple component of the group algebra $Q[G]$ over the rationals $\boldsymbol{Q}$ which corresponds to $\chi$ is canonically isomorphic to the cyclic algebra $\left(-1, \boldsymbol{Q}\left(\zeta_{3}\right) / \boldsymbol{Q}, \iota\right)=\boldsymbol{Q}\left(\zeta_{3}\right)+$ $\boldsymbol{Q}\left(\zeta_{3}\right) u, u^{2}=-1, u \zeta_{3} u^{-1}=\zeta_{3}^{-1}=\ell\left(\zeta_{3}\right)$. This algebra has $\boldsymbol{R}$-local index 2 , and so $m_{R}(\chi)=2$. But 2 does not divide the exponent of $G^{\prime}$ and $2^{3} \nmid|G|$.

THEOREM 5. Let $G$ be a finite group and $\chi$ a complex irreducible character of $G$. Let $p$ be a prime. Suppose $p^{n}(>1)$ divides the Schur index $m_{Q}(\chi)$ of $\chi$ over the rationals $Q$ and $p^{n+1} \nmid m_{Q}(\chi)$. Then either $p^{2 n}$ divides the exponent of $G$ or $p^{n}$ divides the exponent of $G^{\prime}$. If $p^{2 n}$ does not divide the exponent of $G$, then $p^{2 n+1}$ divides the order of $G$. If a Sylow p-subgroup of $G$ is abelian then $p^{2 n}$ divides the exponent of $G$.

Proof. Recall that $m_{Q}(\chi)$ is the least common multiple of the (local) Schur indices $m_{Q_{l}}(\chi)$ and $m_{R}(\chi)$, where $l$ ranges over all the primes. If there is an odd prime $l$ such that $m_{Q_{l}}(\chi)$ is divisible by $p^{n}$, then Theorem 5 is immediate from Theorem 3. If there is no odd prime $l$ with $m_{Q_{l}}(\chi)$ divisible by $p^{n}$, then $p^{n}$ divides either $m_{Q_{2}}(\chi)$ or $m_{R}(\chi)$. It follows that 
$p=2, n=1$. Then by the Fein-Yamada theorem [1], $2^{2}=2^{2 n}$ divides the exponent of $G$, and Theorem 5 is proved.

REMARK. We use the notation of Theorem 5. In [1], we actually proved that either $p^{n+1}$ divides the exponent of $G$ or $p^{n}$ divides the exponent of $G^{\prime}$ (see p. 497 of [1]). The fact that either $p^{2 n}$ divides the exponent of $G$ or $p^{n}$ divides the exponent of $G^{\prime}$ is thus a refinement of part of the Fein-Yamada theorem and was already announced by Ford [2].

\section{References}

[1] B. Fein and T. Yamada, The Schur index and the order and exponent of a finite group, J. Algebra, 28 (1974), 496-498.

[2] C. FoRD, Theorems relating finite groups and division algebras, in Proceedings of the Conference on Finite Groups, ed. by W. Scott, Academic Press, New York, 1976.

[3] E. WItr, Die algebraische Struktur des Gruppenringes einer endlichen Gruppe über einem Zahlkörper, J. Reine Angew. Math., 190 (1952), 231-245.

[4] T. YAMADA, Characterization of the simple components of the group algebras over the $p$-adic number field, J. Math. Soc. Japan, 23 (1971), 295-310.

[5] T. YAMADA, The Schur subgroup of a $p$-adic field, J. Algebra, 31 (1974), 480-498.

[6] T. Yamada, The Schur Subgroup of the Brauer Group, Lecture Notes in Math., Vol. 397, Springer, 1974.

Present Address:

Department of Mathematics

TOKYo Metropolitan UNIVERSITY

Fukazawa, Setagaya-ku, Tokyo 158 